\title{
A Comparative Study between Least-Squares Support Vector Machines and Partial Least Squares in Simultaneous Spectrophotometric Determination of Cypermethrin, Permethrin and Tetramethrin
}

\author{
Ali Niazi,* Mohammad Goodarzi and Ateesa Yazdanipour \\ Department of Chemistry, Faculty of Sciences, Azad University of Arak, Arak, Iran
}

\begin{abstract}
Máquinas de vetor de suporte por mínimos quadrados (LS-SVM) e mínimos quadrados parciais (PLS) têm recebido atenção considerável em quimiometria para análise multicomponente. Foi realizada uma comparação entre os métodos LS-SVM e PLS aplicando-os na determinação espectrofotométrica simultânea de cipermetrina, permetrina e tetrametrina. Os espectros de absorbância no UV de soluções metanólicas foram medidas nas faixas de concentração 0,1-12,9, 0,1-24,9 e $0,1-13,8 \mu \mathrm{g} \mathrm{mL} \mathrm{m}^{-1}$ para cipermetrina, permetrina e tetrametrina, respectivamente. A determinação simultânea por espectrofotometria no UV desses analitos representa um problema em química analítica devido às interferências espectrais. Dessa forma foi realizada a resolução da mistura por métodos quimiométricos. As raízes quadradas do erro quadrático médio de previsão (RMSEP) para cipermetrina, permetrina e tetrametrina por PLS e LS-SVM foram 0,1629, 0,2828, 0,1984 e 0,0766, 0,0907, 0,0467, respectivamente. A habilidade do método para análise de amostras em matrizes sintéticas e reais apresentou resultados satisfatórios. Os dados obtidos mostraram que o LS-SVM produziu melhores resultados que o PLS.
\end{abstract}

Least-squares support vector machines (LS-SVM) and partial least squares (PLS) have received considerable attention in the chemometrics for multicomponent analysis. A comparison was made between LS-SVM and PLS methods by applying them to simultaneous spectrophotometric determination of cypermethrin, permethrin and tetramethrin. The UV absorbance spectra of the methanolic solutions of the analytes were measured in the concentration ranges, 0.1-12.9, 0.1-24.9 and 0.1-13.8 $\mu \mathrm{g} \mathrm{mL} \mathrm{m}^{-1}$ for cypermethrin, permethrin and tetramethrin, respectively. The simultaneous determination of cypermetrin, permetrin and tetrametrin by UV spectrophotometry is a difficult problem in analytical chemistry, due to spectra interferences. The resolution of mixture of cypermetrin, permetrin and tetrametrin by chemometrics methods was performed. The root means square error of prediction (RMSEP) for cypermetrin, permetrin and tetrametrin by PLS and LS-SVM models were $0.1629,0.2828,0.1984$ and $0.0766,0.0907,0.0467$, respectively. The capability of the method for the analysis of synthesis and real matrix samples were evaluated by the determination of cypermethrin, permethrin and tetramethrin with satisfactory results. The resultant data explained that LS-SVM produced better results than PLS.

Keywords: cypermethrin, permethrin, tetramethrin, spectrophotometric, least squares-support vector machines, partial least squares

\section{Introduction}

The simultaneous determination of several components in a mixture can be difficult task, especially when their analytical characteristics are not very different. In recent years attention has been directed toward methods of analysis

*e-mail: ali.niazi@gmail.com involving multicomponent systems based on chemometrics methods. ${ }^{1}$ Nowadays, multivariate calibration has become an indispensable part of modern analytical chemistry. A goal of multicomponent spectral analysis is to construct a calibration model relating the outputs of multivariate spectrometer to the compositions or properties of analytical samples. Among the different regression methods existed for multivariate calibration, the factor analysis-based 
methods including partial least squares (PLS) regression has received considerable attention in the chemometrics literatures..$^{1-3}$ It is based on linear models and is used as satisfactory solution in most cases, where a linear relationship is present between the analytical signal and the property to be determined. However, PLSR is not always the best option, especially in situations where a nonlinear model is clearly required. Theory and application of PLSR have been discussed in previous studies. ${ }^{4-8}$

Support vector machine (SVM) introduced by Vapnik ${ }^{9,10}$ is a valuable tool for solving pattern recognition and classification problem. SVMs can be applied to regression problems by the introduction of an alternative loss function. Due to its advantages and remarkable generalization performance over other methods, SVM has attracted attention and gained extensive application. ${ }^{9}$ SVM shows outstanding performances because it can lead to global models that are often unique by embodies the structural risk minimization principle, ${ }^{9}$ which has been shown to be superior to the traditional empirical risk minimization principle. Furthermore, due to their specific formulation, sparse solutions can be found, and both linear and nonlinear regression can be performed. However, finding the final SVM model can be computationally very difficult because it requires the solution of a set of nonlinear equations (quadratic programming problem). As a simplification, Suykens and Vandewalle ${ }^{11}$ proposed a modified version of SVM called least-squares SVM (LS-SVM), which resulted in a set of linear equations instead of a quadratic programming problem, which can extend the applications of the SVM. There exist a number of excellent introductions of SVM, ${ }^{12-24}$ and the theory of LS-SVM has also been described clearly by Suykens et al. ${ }^{11,12}$ and application of LS-SVM in quantification and classification reported by some of the workers. ${ }^{25-32}$ So, we will only briefly describe the theory of LS-SVM.

In principle, LS-SVM always fits a linear relation $(y=w x+b)$ between the regression $(x)$ and the dependent variable $(y)$. The best relation is the one that minimizes the cost function $(Q)$ containing a penalized regression error term:

$Q=\frac{1}{2} w^{T} w+\frac{1}{2} \gamma \sum_{i=1}^{N} e_{i}^{2}$

subject to:

$y_{i}=w^{T} \phi\left(x_{i}\right)+b+e_{i} \quad i=1, \ldots, N$,

where $\phi$ denotes the feature map. The first part of this cost function is a weight decay which is used to regularize weight sizes and penalize large weights. Due to this regularization, the weights converge to similar value. Large weights deteriorate the generalization ability of the LS-SVM because they can cause excessive variance. The second part of cost function is the regression error for all training data. The relative weight of this part as compared to the first part indicated by the parameter $\gamma$, which has to be optimized by the user.

Similar to other multivariate statistical models, the performances of LS-SVMs depends on the combination of several parameters. The attainment of the kernel function is cumbersome and it will depend on each case. However, the kernel function more used is the radial basis function (RBF), $\exp \left(-\left(\left\|x_{i}-x_{j}^{2}\right\|\right) / 2 \sigma^{2}\right)$ a simple Gaussian function, and polynomial functions $\left\langle x_{i}, x_{i}\right\rangle^{d}$ where $\sigma^{2}$ is the width of the Gaussian function and $d$ is the polynomial degree, which should be optimized by the user, to obtain the support vector. For $\sigma$ of the RBF kernel and $d$ of the polynomial kernel it should be stressed that it is very important to do a careful model selection of the tuning parameters, in combination with the regularization constant $\gamma$, in order to achieve a good generalization model.

Pyrethroid insecticides are used to control a number of insect species on economic crops. Pyrethroids are effective pest control chemicals and have low mammalian toxicity. Cypermetrin, permetrin and tetrametrin have been identified as highly effective contact insecticides. Owing to its availability, insecticides are misused in homicidal/ suicidal poisoning cases. Consequently, detection and determination of these insecticides is necessary in forensic toxicology. ${ }^{33,34}$ In this project, least-squares support vector machines in conjugation with UV absorbance was used to simultaneous determination of cypermethrin, permethrin and tetramethrin in synthetic and real samples. A comparison was also made between LS-SVM and PLS regression to extent the approach existed in the filed. The proposed methodology is fast, simple and does not generate hazardous chemical wastes, thus makes it easily possible to be used in control analysis of cypermethrin, permethrin and tetramethrin in environmental.

\section{Experimental}

\section{Reagents and solutions}

All reagents were of analytical reagent grade. Stock standard solutions of cypermethrin, permethrin and tetramethrin, $1000 \mu \mathrm{g} \mathrm{mL}-1$, were prepared by dissolving appropriate amount of solutes in methanol. Working solutions of lower concentrations were prepared by proper dilution with methanol from the stock standard solutions. This solution was stored in the dark at $4{ }^{\circ} \mathrm{C}$ and was found to be stable for at least 2 weeks. 


\section{Apparatus and software}

A Hewlett-Packard 8453 diode array spectrophotometer controlled by a Hewlett-Packard computer and equipped with a 1-cm path length quartz cell was used for UV spectra acquisition. Data acquisition between 200 and $260 \mathrm{~nm}$ were performed with UV-Visible ChemStation program (Agilent Technologies), running under Windows XP.

The quantitative evaluations were carried out by using the PLS program from PLS-Toolbox version 2.0 for use with Matlab from Eigenvector Research Inc. The LS-SVM optimization and model results were obtained using the LS-SVM lab toolbox (Matlab/C Toolbox for Least-Squares Support Vector Machines). ${ }^{11}$ All programs were run on a Pentium IV (CPU at 3.0 GHz and RAM 1.0 GB) personal computer with windows XP operating system was used.

\section{Procedure}

\section{Standard calibration and prediction set}

A mixture design was used to maximize statistically the information content in the spectra. ${ }^{35}$ According to 21 experimental design (Table 1) solutions were used to construct the models (calibration set) and another 10 solutions to validate them (prediction set) in that these were not included in the calibration set but were employed for independent tests (Table 2). The concentrations of cypermethrin, permethrin and tetramethrin were varied between 0.1-12.9, 0.1-24.9 and 0.1-13.8 $\mu \mathrm{g} \mathrm{mL}^{-1}$, respectively. The mixed standard solutions were placed in a $10 \mathrm{~mL}$ volumetric flask and completed to the final volume with methanol. The absorption spectra were recorded between 200 and $260 \mathrm{~nm}$ against blank of methanol. The spectra region between 200 and $260 \mathrm{~nm}$, which implies working with 61 experimental points for each spectra (as the spectra are digitized each $1.0 \mathrm{~nm}$ ), was selected for analysis, because this is the zone with the maximum spectral information from the mixture components of interest.

Procedure for the determination of cypermethrin, permethrin and tetramethrin in groundwater

After addition amounts of cypermethrin, permethrin and tetramethrin to water samples, double extraction with n-hexane was used as below. Water samples

Table 1. Concentration data of the different mixtures used in the calibration set for the determination of cypermethrin, permethrin and tetramethrin $\left(\mu \mathrm{g} \mathrm{mL} L^{-1}\right)$

\begin{tabular}{lccc}
\hline Mixture & Cypermethrin & Permethrin & Tetramethrin \\
\hline M1 & 0.1 & 0.1 & 13.8 \\
M2 & 2.7 & 0.1 & 10.9 \\
M3 & 5.3 & 0.1 & 8.2 \\
M4 & 7.9 & 0.1 & 5.5 \\
M5 & 10.5 & 0.1 & 2.8 \\
M6 & 12.9 & 0.1 & 0.1 \\
M7 & 10.5 & 5.1 & 0.1 \\
M8 & 7.9 & 10.1 & 0.1 \\
M9 & 5.3 & 15.1 & 0.1 \\
M10 & 2.7 & 20.1 & 0.1 \\
M11 & 0.1 & 24.9 & 0.1 \\
M12 & 0.1 & 20.1 & 2.8 \\
M13 & 0.1 & 15.1 & 5.5 \\
M14 & 0.1 & 10.1 & 8.2 \\
M15 & 0.1 & 5.1 & 10.9 \\
M16 & 2.7 & 5.1 & 8.2 \\
M17 & 5.3 & 5.1 & 5.5 \\
M18 & 7.9 & 5.1 & 2.8 \\
M19 & 2.7 & 10.1 & 5.5 \\
M20 & 5.3 & 10.1 & 2.8 \\
M21 & 2.7 & 15.1 & 2.8 \\
\hline
\end{tabular}

Table 2. Composition of synthetic mixtures and predicted values for determination of cypermethrin, permethrin and tetramethrin $\left(\mu \mathrm{g} \mathrm{mL}^{-1}\right)$

\begin{tabular}{cccccccccccccccccc}
\hline & Added & \multicolumn{4}{c}{ Found (PLS) } & \multicolumn{3}{c}{ Error (\%) } & \multicolumn{3}{c}{ Found (LS-SVM) } & \multicolumn{3}{c}{ Error (\%) } \\
\hline Cyp. & Per. & Tet. & Cyp. & Per. & Tet. & Cyp. & Per. & Tet. & Cyp. & Per. & Tet. & Cyp. & Per. & Tet. \\
0.5 & 3.5 & 5.0 & 0.53 & 3.72 & 5.08 & 6.00 & 6.29 & 1.60 & 0.51 & 3.53 & 4.99 & 2.00 & 0.75 & -0.16 \\
5.5 & 10.0 & 3.0 & 5.62 & 9.23 & 2.97 & 2.18 & -7.70 & -0.92 & 5.52 & 9.75 & 3.07 & 0.36 & -2.53 & 2.35 \\
0.3 & 2.0 & 11.0 & 0.29 & 2.11 & 10.56 & -4.94 & 5.50 & -4.00 & 0.31 & 2.03 & 10.93 & 3.33 & 1.53 & -0.68 \\
8.0 & 0.3 & 2.5 & 7.83 & 0.28 & 2.64 & -2.09 & -6.67 & 5.60 & 7.95 & 0.29 & 2.54 & -0.68 & -3.33 & 1.78 \\
2.0 & 2.0 & 2.0 & 2.09 & 1.89 & 1.91 & 4.50 & -5.50 & -4.50 & 2.02 & 2.05 & 2.03 & 1.05 & 2.55 & 1.38 \\
4.2 & 3.5 & 0.3 & 4.34 & 3.34 & 0.29 & 3.44 & -4.57 & -3.33 & 4.20 & 3.53 & 0.30 & -0.02 & 0.97 & 0.00 \\
6.5 & 12.0 & 10.0 & 6.53 & 11.87 & 9.74 & 0.50 & -1.07 & -2.59 & 6.44 & 12.03 & 9.94 & -0.97 & 0.23 & -0.57 \\
11.5 & 2.0 & 5.0 & 11.10 & 2.08 & 4.94 & -3.48 & 4.00 & -1.20 & 11.28 & 2.02 & 4.94 & -1.92 & 1.00 & -1.25 \\
3.8 & 18.0 & 6.5 & 3.94 & 17.74 & 6.61 & 3.68 & -1.46 & 1.75 & 3.81 & 17.90 & 6.48 & 0.16 & -0.58 & -0.27 \\
2.7 & 16.0 & 8.2 & 2.59 & 16.13 & 7.91 & -4.10 & 0.81 & -3.48 & 2.74 & 15.97 & 8.17 & 1.57 & -0.18 & -0.36 \\
\hline
\end{tabular}


$(500 \mathrm{~mL}$ ) were shaken with $50 \mathrm{~mL}$ of $n$-hexane for $2 \mathrm{~min}$. The collected organic phases were dried by passing them through anhydrous $\mathrm{Na}_{2} \mathrm{SO}_{4}$ and evaporated using a rotary vacuum evaporator. The concentrated samples were eluted with $1 \mathrm{~mL}$ acetonitrile and cypermethrin, permethrin and tetramethrin were determined as described above.

\section{Statistical parameters}

For the evaluation of the predictive ability of a multivariate calibration model, the root mean square error of prediction (RMSEP) can be used:

$R M S E P=\sqrt{\frac{\sum_{i=1}^{n}\left(y_{\text {pred }}-y_{\text {obs }}\right)^{2}}{n}}$

where $y_{\text {pred }}$ is the predicted concentration in the sample, $y_{\text {obs }}$ is the observed value of the concentration in the sample and $n$ is the number of samples in the validation set. The square of correlation coefficient $\left(\mathrm{R}^{2}\right)$, which indicates the fitting of data in a straight line, was calculated as:

$R^{2}=\frac{\sum_{i=1}^{n}\left(y_{\text {pred }}-\bar{y}\right)^{2}}{\sum_{i=1}^{n}\left(y_{o b s}-\bar{y}\right)^{2}}$

where $\bar{y}$ is the mean of actual concentration in the prediction set.

\section{Selection of optimum number of factors}

The selection of the number of factors in the factor analysis-based methods is very important for achieving the best prediction. The model refinement procedure used the predicted residual errors sum of squares (PRESS) of the leave-one-out cross-validation (LOO-CV) to select the optimal number of PLS factors. The PRESS was computed, which is defined as follows:

PRESS $=\sum\left(y_{i}-\hat{y}_{i}\right)^{2}$

where $y_{i}$ is the reference concentration for the $i$ th sample and $\hat{y}_{i}$ represents the estimated concentration. To select the optimum number of factors, the criterion of Haaland and Thomas ${ }^{36}$ was used. The maximum number of factors used to determine the optimum PRESS was selected as 11 (half the number of standards plus one). The $F$-statistic was used to make the significance determination. As the difference between the minimum PRESS and the other PRESS values becomes smaller, the probability that each additional factor is significant also decreases. Haaland and
Thomas empirically determined that an $F$-ratio probability of 0.75 is a good choice. We selected as the optimum the number of factors for the first PRESS value whose $F$-ratio probability drops below 0.75 .

\section{Results and Discussion}

Figure 1 shows the absorption spectra for the individual methanolic solution of cypermethrin, permethrin and tetramethrin. As this figure shows, there is a clear overlapping of the three spectra. This prevents the simultaneous determination of the cypermethrin, permethrin and tetramethrin by direct UV absorbance measurements. This problem was overcome by the use of least-squares support vector machines.

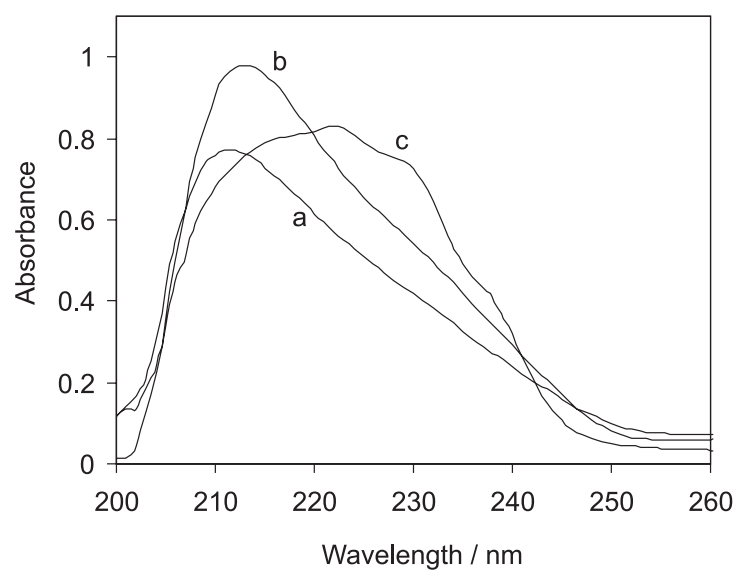

Figure 1. Absorption spectra of $8.5 \mu \mathrm{g} \mathrm{mL}^{-1}$ methanolic solutions of the (a) cypermethrin, (b) permethrin and (c) tetramethrin used in this study.

\section{Mixture design}

The standard solutions used in the multivariate calibration methods are mixtures of analytes. The quality of the multivariate calibration methods is dependent on the standard mixture solutions and presence or absence of outliers. Some important parameters should be inserted in preparing these standard solutions. First, the concentration of each analyte must be in its linear dynamic range; second, the concentration of analytes in the calibration samples must be orthogonal in order to give the most information from the analytical system; third, the sum of the concentrations of the analytes in a given mixture must be so that its absorbance does not exceed the maximum absorbance reading of the instrument; finally, the concentration of the prediction mixtures should span the same space as that of calibration mixtures.

Individual calibration curves were constructed with several points as absorbance versus cypermethrin, 
permethrin and tetramethrin concentrations. For constructing the individual calibration lines, the absorbencies were measured at 212, 214 and $222 \mathrm{~nm}$ against a blank for cypermethrin, permethrin and tetramethrin, respectively. The linear regression equation for the calibration graph for cypermethrin for the concentration range of $0.1-12.9 \mu \mathrm{g} \mathrm{mL}^{-1}$ was $\mathrm{A}=0.0462+0.0852 \mathrm{C}$ $\left(r^{2}=0.9912, n=16\right)$, for permethrin for the concentration range $0.1-24.9 \mu \mathrm{g} \mathrm{mL}^{-1}$ was $\mathrm{A}=0.0840+0.0634 \mathrm{C}_{\text {permethrin }}$ $\left(r^{2}=0.9956, n=16\right)$ and for tetramethrin for the concentration range $0.1-13.8 \mu \mathrm{g} \mathrm{mL}^{-1}$ was $\mathrm{A}=0.0115+$ $0.0499 \mathrm{C}_{\text {tetramethrin }}\left(\mathrm{r}^{2}=0.9925, \mathrm{n}=15\right)$. The limits of detection were $0.04,0.07$ and $0.05 \mu \mathrm{g} \mathrm{mL}^{-1}$ for cypermethrin, permethrin and tetramethrin, respectively, and these were calculated according to calibration line characteristics.

According to procedure section, the calibration matrix was designed. In Table 1, the compositions of the ternary mixtures used in the calibration matrices are summarized. For prediction set, ten mixtures were prepared according to procedure section (see Table 2). Principal component analysis (PCA) was used to check the standard mixtures for the presence of outlier and also to ensure the homogeneity between the calibration and prediction samples. To do so, the absorbance data matrices of the calibration and prediction samples were subjected to PCA, separately, and the standard mixtures were plotted in the factor space.

\section{Partial least squares analysis}

Firstly, the PLS regression was applied on the spectral data for simultaneous determination of the analytes. Leaveone-out cross-validation procedure was used to select the optimum number of PLS-latent variables for each analyte and the number of factors that produced the least PRESS was selected as optimum value. A plot of PRESS for cypermetrin, permetrin and tetrametrin as a function of the number of factors is shown in Figure 2. Table 3 shows the optimum

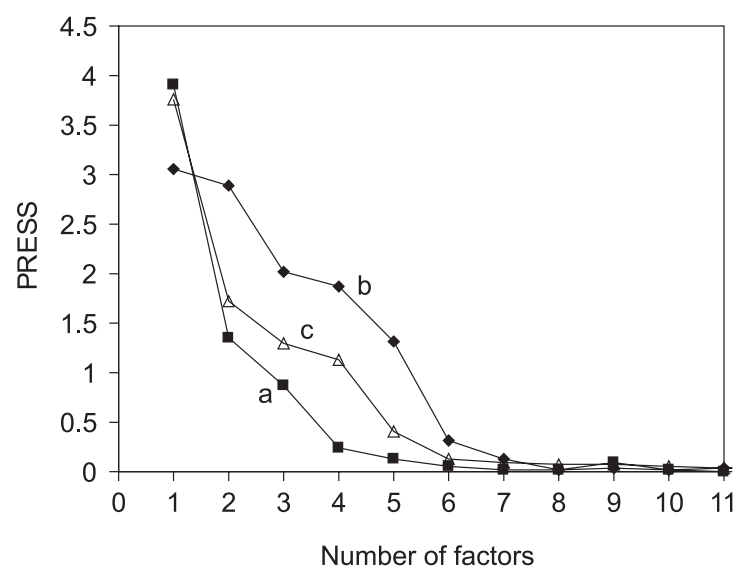

Figure 2. Plots of PRESS of cross-validation for (a) cypermethrin, (b) permethrin and (c) tetramethrin as a function of the number of factors.

number of factors and PRESS values for cypermetrin, permetrin and tetrametrin. The number of PLS-latent variables used to model absorbance-concentration is higher than the number of analytes, which can be attributed to the interaction between the compounds in the mixtures.

To investigate the prediction ability of the resulted PLS model and to compare with least-squares support vector machines, the calibrated PLS model were used for quantization of the analytes in a separate prediction set mixtures that did not have contribution in the model building steps. The resulted predicted concentrations along with the statistical quantities are represented in Table 2 and 3.

\section{Least-squares support vector machines}

LS-SVM was performed with radial basis function (RBF) as a kernel function. In the model development using LS-SVM and RBF kernel, $\gamma$ and $\sigma^{2}$ parameters were a manageable task, similar to the process employed to select the number of factors for PLS models, but in this case for a two-dimensional problem. In Figure 3 is

Table 3. Statistical parameters for the PLS and LS-SVM calibration models

\begin{tabular}{|c|c|c|c|c|c|c|}
\hline \multirow{2}{*}{$\begin{array}{l}\text { Method } \\
\text { Compound }\end{array}$} & \multicolumn{3}{|c|}{ PLS } & \multicolumn{3}{|c|}{ LS-SVM } \\
\hline & Cypermethrin & Permethrin & Tetramethrin & Cypermethrin & Permethrin & Tetramethrin \\
\hline $\mathrm{NF}^{\mathrm{a}}$ & 5 & 6 & 5 & & & \\
\hline PRESS & 0.1230 & 0.3214 & 0.4223 & & & \\
\hline$\gamma$ & & & & 100 & 100 & 100 \\
\hline$\sigma^{2}$ & & & & 10 & 10 & 10 \\
\hline RMSEP & 0.1629 & 0.2828 & 0.1984 & 0.0766 & 0.0907 & 0.0467 \\
\hline $\mathrm{R}^{2}$ & 0.9401 & 0.9748 & 0.9267 & 0.9631 & 0.9861 & 0.9799 \\
\hline $\operatorname{LOD}\left(\mu \mathrm{g} \mathrm{mL}^{-1}\right)^{\mathrm{b}}$ & 0.065 & 0.057 & 0.071 & 0.042 & 0.038 & 0.046 \\
\hline
\end{tabular}

${ }^{a}$ Number of factors; ${ }^{b} \mathrm{LOD}$ (limit of detection) $=3.3 \mathrm{~s}(0)$ where $\mathrm{s}(0)$ is the S.D. in the predicted concentration of cypermethrin, permethrin and tetramethrin in a blank sample ${ }^{37}$. 
shown the optimized surface result for the LS-SVM and RBF kernel, using the spectra calibration sets for three pyrethroid insecticides. These parameters were optimized generating models with values of $\gamma$ in the range of 1-500 and $\sigma^{2}$ in the range of 1-100 with adequate increments. These ranges were selected from previous studies where it was establish the magnitude of the parameters to be optimized. For each combination of $\gamma$ and $\sigma^{2}$ parameters, root mean square error of cross-validation (RMSECV) was calculated and the optimum parameters were selected produced the smaller RMSECV. The cross-validation procedure was used to determine an average RMSECV between the three RMSCEV of the components, using the following equation:

$R M S E C V=\frac{\text { MMSECV }_{\text {cypermecthin }}+\mathrm{RMSECV}_{\text {permethrin }}+\mathrm{RMSECV}_{\text {teramechrin }}}{3}(6)$

In Table 3 the optimum $\gamma$ and $\sigma^{2}$ parameters for the LS-SVM and RBF kernel are shown, using the calibration set for ternary mixtures of cypermethrin, permethrin and tetramethrin. Table 3 also shows RMSEP and $\mathrm{R}^{2}$ for comparison.

Determination of cypermethrin, permethrin and tetramethrin in synthetic and real samples

The proposed method was successfully applied for determination of cypermethrin, permethrin and tetramethrin in several synthetic samples (their compositions are given in Table 2). The results obtained from simultaneous determination of cypermethrin, permethrin and tetramethrin by PLS and LS-SVM methods are listed in Table 2 and 3. Table 2 also shows the percentage error for synthetic series of cypermethrin, permethrin and tetramethrin mixtures. As can be seen, the percentage error was also quite acceptable. Good results were achieved in LS-SVM model with percentage error ranges from -1.92 to $3.33 \%$, -3.33 to $2.55 \%$ and -1.25 to $2.35 \%$ for cypermethrin, permethrin and tetramethrin, respectively. Figures of merit are regularly employed for method comparison. The results for the determination of these three substances and their reported figure of merits are reported in the Table 3. Table 3 also shows the RMSEP and $\mathrm{R}^{2}$ for synthetic series of cypermethrin, permethrin and tetramethrin mixtures.

The proposed procedure was successfully applied for the assay of cypermethrin, permethrin and tetramethrin simultaneously in real samples. For this purpose, diverse spiked samples and reference materials were analyzed. Samples were treated and analyzed as described under procedure section. Table 4 summarizes the results obtained for real matrix samples. Table 4 shows that satisfactory recovery values were obtained for the samples assayed. Therefore, the LS-SVM model is able to predict the concentrations of cypermethrin, permethrin and tetramethrin in the real matrix sample.

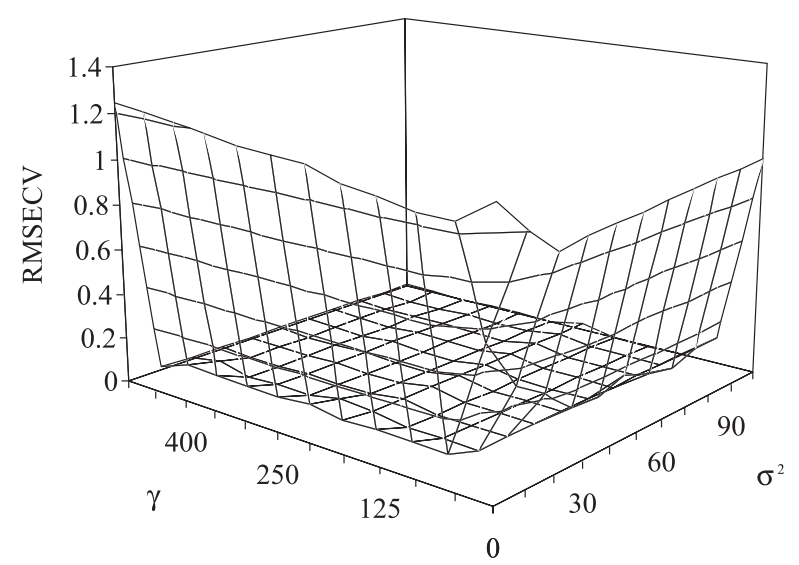

Figure 3. Parameter optimization response surface for LS-SVM model.

\section{Conclusion}

PLS and LS-SVM as two powerful and the most widely used multivariate calibration methods were employed for simultaneous spectrophotometric determination of cypermethrin, permethrin and tetramethrin. The cypermethrin, permetrin and tetramethrin mixture is an extremely difficult complex system due to the high spectra overlapping observed between the spectra for

Table 4. PLS and LS-SVM results applied on the real matrix samples $\left(\mu \mathrm{g} \mathrm{mL}^{-1}\right)$

\begin{tabular}{|c|c|c|c|c|c|c|c|c|c|c|c|c|}
\hline \multirow{3}{*}{ Samples } & \multicolumn{4}{|c|}{ Cypermethrin } & \multicolumn{4}{|c|}{ Permethrin } & \multicolumn{4}{|c|}{ Tetramethrin } \\
\hline & \multicolumn{2}{|c|}{ Determined } & \multicolumn{2}{|c|}{ Recovery (\%) } & \multicolumn{2}{|c|}{ Determined } & \multicolumn{2}{|c|}{ Recovery (\%) } & \multicolumn{2}{|c|}{ Determined } & \multicolumn{2}{|c|}{ Recovery (\%) } \\
\hline & PLS & LS-SVM & PLS & LS-SVM & PLS & LS-SVM & PLS & LS-SVM & PLS & LS-SVM & PLS & LS-SVM \\
\hline $1^{\text {(a) }}$ & 0.93 & 0.99 & 93.0 & 99.0 & 1.81 & 1.97 & 90.5 & 98.5 & 2.13 & 2.02 & 106.5 & 101.0 \\
\hline $2^{\text {(b) }}$ & 4.46 & 4.97 & 89.2 & 99.4 & 4.19 & 4.11 & 104.8 & 102.8 & 4.29 & 4.05 & 107.3 & 101.3 \\
\hline $3^{\text {(c) }}$ & 2.63 & 2.56 & 105.2 & 102.4 & 2.85 & 2.94 & 95.0 & 98.0 & 5.16 & 5.03 & 103.2 & 100.6 \\
\hline
\end{tabular}

Added of cypermethrin, permethrin and tetramethrin: (a) 1.0, 2.0 and $2.0 \mu \mathrm{g} \mathrm{mL}{ }^{-1}$, (b) $5.0,4.0$ and $4.0 \mu \mathrm{g} \mathrm{mL} \mathrm{md}^{-1} .5,3.0$ and $5.0 \mu \mathrm{g} \mathrm{mL} \mathrm{an}^{-1}$, respectively. 
their components. For overcoming the drawback of spectrophotometric interferences, PLS and LS-SVM multivariate calibration approaches are applied. According to the obtained results (percentage of error, RMSEP, $\mathrm{R}^{2}$ and figure of merit), LS-SVM is better than PLS. LS-SVM gives a low limit of detection. The results of this study clearly show the potential and versatility of this method, which could be applied to simultaneous determination of cypermethrin, permethrin and tetramethrin in synthetic and real matrix samples.

\section{Acknowledgment}

The authors gratefully acknowledge the support to this work from Azad University of Arak research council, especially Mr. H. Malekhossieni for preparation of reagents.

\section{References}

1. Martens, M.; Naes, T.; Multivariate Calibration, Wiley: Chichester, 1989.

2. Geladi, P.; Kowalski, B. R.; Anal. Chim. Acta 1986, 185, 1.

3. Brereton, R. G.; Chemometrics; Data Analysis for the Laboratory and Chemical Plant, Wiley: England, 2003.

4. Niazi, A.; Ghasemi, J.; Yazdanipour, A.; Anal. Lett. 2005, 38, 2377.

5. Niazi, A.; Soufi, A.; Mobarakabadi, M.; Anal. Lett. 2006, 39, 2359.

6. Niazi, A.; Sadeghi, M.; Chem. Pharm. Bull. 2006, 54, 711.

7. Niazi, A.; J. Braz. Chem. Soc. 2006, 17, 1020.

8. Niazi, A.; Croa. Chim. Acta 2006, 79, 573.

9. Vapnik, V.; Statistical Learning Theory, John Wiley: New York, 1998.

10. Cortes, C.; Vapnik, V.; Mach. Learn. 1995, 20, 273.

11. Suykens, J. A. K.; Vandewalle, J.; Neural Process. Lett. 1999, 9, 293.

12. Suykens, J. A. K.; van Gestel, T.; de Brabanter, J.; de Moor, B.; Vandewalle, J.; Least-Squares Support Vector Machines, World Scientifics: Singapore, 2002.

13. Vapnik, V.; In Nonlinear Modeling: Advanced Black-Box Techniques; Suykens, J. A. K.; Vandewalle, J., eds.; Kluwer Academic Publishers: Boston, 1998.

14. Suykens, J. A. K.; Eur. J. Control 2001, 7, 311.

15. Burges, C. J. C.; Data Min. Knowl. Discov. 1998, 2, 121.
16. Smola, A. J.; Scholkopf, B.; Stat. Comput. 2004, 14, 199.

17. Zomer, S.; Brereton, R. G.; Carter, J. F.; Eckers, C.; Analyst 2004, 129, 175.

18. Belousov, A. I.; Verzakov, S. A.; Frese, J. V.; J. Chemom. 2002, $16,482$.

19. Pierna, J. A. F.; Volery, P.; Besson, R.; Baeten, V.; Dardenne, P.; J. Agric. Food. Chem. 2005, 53, 6581.

20. Thissen, U.; Ustun, B.; Melssen, W. J.; Buydens, L. M. C.; Anal. Chem. 2004, 76, 3099.

21. Chauchard, F.; Cogdill, R.; Roussel, S.; Roger, J. M.; BellonMaurel V.; Chemom. Intell. Lab. Syst. 2004, 71, 141.

22. Thissen, U.; van Brakel, R.; de Weijer, A. P.; Melssen, W. J.; Buydens, L. M. C.; Chemom. Intell. Lab. Syst. 2003, 69, 35.

23. Zhao, J.; Chen, Q.; Huang, X.; Fang, C. H.; J. Pharm. Biomed. Anal. 2006, 41, 1198.

24. Zou, T.; Dou, Y.; Mi, H.; Zou, J.; Ren, Y.; Anal. Biochem. 2006, 355,1 .

25. Ke, Y.; Yiyu, C.; Chinese J. Anal. Chem. 2006, 34, 561.

26. Borin, A.; Ferrao, M. F.; Mello, C.; Maretto, D. A.; Poppi, R. J.; Anal. Chim. Acta 2006, 579, 25.

27. Kovalenko, I. V.; Rippke, G. R.; Hurburgh, C. R.; J. Agric. Food. Chem. 2006, 54, 3485.

28. Kovalenko, I. V.; Rippke, G. R.; Hurburgh, C. R.; J. Am. Oil Chem. Soc. 2006, 85, 421.

29. Li, J.; Liu, H.; Yao, X.; Liu, M.; Hu, Z.; Fan, B.; Anal. Chim. Acta 2007, 581, 333.

30. Arfken, G. B.; Weber, H. J.; Mathematical Methods for Physicists, $4^{\text {th }}$ ed., Academic Press: New York, 1995.

31. Li, Y.; Shao, X.; Cai, W.; Talanta 2007, 72, 217.

32. Niazi, A.; Ghasemi, J.; Yazdanipour, A.; Spectrochim. Acta Part A 2007, 68, 523.

33. Vitthal, B. P.; Murlidhar, T. S.; Sudhakar, V. P.; Analyst 1992, 117,75 .

34. Janghel, E. K.; Rai, J. K.; Rai, M. K.; Gupta, V. K.; J. Braz. Chem. Soc. 2007, 18, 590.

35. Cornel, J. A.; Experiments with Mixtures, Wiley: New York, 1981.

36. Haaland, D. M.; Thomas, E. V.; Anal. Chem. 1988, 60, 1193.

37. Boque, R.; Ferre, J.; Faber, N. M.; Rius, F. X.; Anal. Chim. Acta 2002, 451, 313.

Received: August 2, 2007

Web Release Date: March 13, 2008 